\title{
External Ellipsoidal Harmonics for the Dunkl-Laplacian ${ }^{\star}$
}

\author{
Hans VOLKMER
}

Department of Mathematical Sciences, University of Wisconsin-Milwaukee, P. O. Box 413, Milwaukee, WI 53201, USA

E-mail:volkmer@uwm.edu

URL: http: //www. uwm.edu/ volkmer/

Received September 22, 2008, in final form December 18, 2008; Published online December 23, 2008 Original article is available at http://www.emis.de/journals/SIGMA/2008/091/

\begin{abstract}
The paper introduces external ellipsoidal and external sphero-conal $h$-harmonics for the Dunkl-Laplacian. These external $h$-harmonics admit integral representations, and they are connected by a formula of Niven's type. External $h$-harmonics in the plane are expressed in terms of Jacobi polynomials $P_{n}^{\alpha, \beta}$ and Jacobi's functions $Q_{n}^{\alpha, \beta}$ of the second kind.
\end{abstract}

Key words: external ellipsoidal harmonics; Stieltjes polynomials; Dunkl-Laplacian; fundamental solution; Niven's formula; Jacobi's function of the second kind

2000 Mathematics Subject Classification: 33C52; 35C10

\section{Introduction}

In a previous article [21] the author introduced (internal) ellipsoidal $h$-harmonics $F_{\mathbf{n}, \mathbf{p}}$ and sphero-conal $h$-harmonics $G_{\mathbf{n}, \mathbf{p}}$ for the Dunkl-Laplacian. The functions $F_{\mathbf{n}, \mathbf{p}}(\mathbf{x})$ and $G_{\mathbf{n}, \mathbf{p}}(\mathbf{x})$ are polynomials in the variables $\mathbf{x}=\left(x_{0}, x_{1}, \ldots, x_{k}\right)$ and they are $h$-harmonic, that is, they satisfy the differential-difference equation

$$
\Delta_{h} u:=\left(\mathcal{D}_{0}^{2}+\mathcal{D}_{1}^{2}+\cdots+\mathcal{D}_{k}^{2}\right) u=0,
$$

where $\mathcal{D}_{j}$ is defined by

$$
\mathcal{D}_{j} u(\mathbf{x}):=\frac{\partial u(\mathbf{x})}{\partial x_{j}}+\alpha_{j} \frac{u(\mathbf{x})-u\left(\sigma_{j}(\mathbf{x})\right)}{x_{j}}
$$

and $\sigma_{j}$ is the reflection at the $j$ th coordinate plane. The parameters $\alpha_{j}$ enter the weight function

$$
h(\mathbf{x}):=\left|x_{0}\right|^{\alpha_{0}}\left|x_{1}\right|^{\alpha_{1}} \cdots\left|x_{k}\right|^{\alpha_{k}} .
$$

Following the book [9] by Dunkl and Xu and the paper [23] by $\mathrm{Xu}$ we will assume that $\alpha_{j} \geq 0$ for each $j=0,1, \ldots, k$ although one would expect that the range of validity can be extended analytically to the domain $\alpha_{j}>-\frac{1}{2}$ for each $j$. We will also exclude the case $k=1, \alpha_{0}=\alpha_{1}=0$ because we want the constant $\mu$ defined below in (1.5) to be positive.

The parity vector $\mathbf{p}=\left(p_{0}, p_{1}, \ldots, p_{k}\right)$ has components in $\{0,1\}$ and indicates that $F_{\mathbf{n}, \mathbf{p}}$ and $G_{\mathbf{n}, \mathbf{p}}$ have parity $\mathbf{p}$ which means that they are sums of monomials $x_{0}^{p_{0}+2 q_{0}} x_{1}^{p_{1}+2 q_{1}} \cdots x_{k}^{p_{k}+2 q_{k}}$ with $q_{j} \in \mathbb{N}_{0}=\{0,1,2, \ldots\}$. The vector $\mathbf{n}=\left(n_{1}, n_{2}, \ldots, n_{p}\right)$ counts the zeros of the corresponding Stieltjes quasi-polynomials inside $k$ adjacent open intervals. If we set $m=2|\mathbf{n}|+|\mathbf{p}|:=$

\footnotetext{
${ }^{\star}$ This paper is a contribution to the Special Issue on Dunkl Operators and Related Topics. The full collection is available at http://www.emis.de/journals/SIGMA/Dunkl_operators.html
} 
$2\left(n_{1}+\cdots+n_{k}\right)+\left(p_{0}+\cdots+p_{k}\right)$ then $G_{\mathbf{n}, \mathbf{p}}$ is a spherical $h$-harmonic of degree $m$, that is, it is a $h$-harmonic homogeneous polynomial of degree $m$. Moreover, $F_{\mathbf{n}, \mathbf{p}}$ is a polynomial of total degree $m$ which, however, is usually not homogeneous. The sphero-conal $h$-harmonics of fixed degree $m$ form an orthogonal basis in the linear space of spherical $h$-harmonics of degree $m$ with respect to the inner product

$$
\langle u, v\rangle=\int_{\mathbf{S}^{k}} h^{2}(\mathbf{x}) u(\mathbf{x}) v(\mathbf{x}) d \mathbf{S}(\mathbf{x}),
$$

where $\mathbf{S}^{k}$ denotes the unit sphere in $\mathbb{R}^{k+1}$. Equation (1.3) uses the standard surface integral over the sphere so that $\int_{\mathbf{S}^{k}} 1 d \mathbf{S}$ is the surface area of $\mathbf{S}^{k}$.

In the present paper the theory is extended to include external ellipsoidal $h$-harmonics $\mathcal{F}_{\mathbf{n}, \mathbf{p}}$ and external sphero-conal $h$-harmonics $\mathcal{G}_{\mathbf{n}, \mathbf{p}}$. Throughout, we will use calligraphic letters to denote the "external" functions. The functions $\mathcal{G}_{\mathbf{n}, \mathbf{p}}$ and $\mathcal{F}_{\mathbf{n}, \mathbf{p}}$ are still solutions of (1.1) but they are no longer polynomials. They have proper decay as $\|\mathbf{x}\| \rightarrow \infty$ which makes them suitable for solving problems concerning the exterior of ellipsoids or spheres, respectively. The external sphero-conal $h$-harmonics are easy to define. They are given by

$$
\mathcal{G}_{\mathbf{n}, \mathbf{p}}(\mathbf{x}):=\|\mathbf{x}\|^{-2 \mu-2 m} G_{\mathbf{n}, \mathbf{p}}(\mathbf{x}), \quad m:=2|\mathbf{n}|+|\mathbf{p}|,
$$

where

$$
\mu:=\alpha_{0}+\alpha_{1}+\cdots+\alpha_{k}+\frac{k-1}{2} .
$$

The definition of external ellipsoidal $h$-harmonics is postponed to Section 2 .

After introducing the fundamental solution of (1.1) in Section 3 we obtain integral representations of $\mathcal{F}_{\mathbf{n}, \mathbf{p}}$ and $\mathcal{G}_{\mathbf{n}, \mathbf{p}}$ in terms of the fundamental solution in Section 4. Section 5 states some integral formulas related to Section 5.2 in [9]. As a main result of this paper, in Section 6 we prove a Niven type formula that expresses $\mathcal{F}_{\mathbf{n}, \mathbf{p}}$ in terms of $\mathcal{G}_{\mathbf{n}, \mathbf{p}}$. In the classical situation $k=2$, $\boldsymbol{\alpha}=0$, this formula was first given by Niven [16] in an amazing award winning paper. Modified versions of some of Niven's result are contained in the books by Whittaker and Watson [22] and by Hobson [14]. It was the attempt to extend Niven's results to external $h$-harmonics that led to this paper. We also refer to paper [15] by Kalnins and Miller which contains a generalization of Niven's work from a different perspective.

In Section 7 we illustrate the results in the relatively simple but nontrivial planar case $k=1$. In this situation the internal ellipsoidal and sphero-conal $h$-harmonics are expressed in terms of Jacobi polynomials $P_{n}^{(\alpha, \beta)}$ while the external ellipsoidal $h$-harmonic $\mathcal{F}_{\mathbf{n}, \mathbf{p}}$ involves the second solution $Q_{n}^{(\alpha, \beta)}$ of the differential equation whose first solution is $P_{n}^{(\alpha, \beta)}$.

We should mention that there is an increasing number of papers in Applied Mathematics that use ellipsoidal harmonics; for example, see [3, 4, 5]. For applications of Dunkl operators and the associated Laplacian to mathematical physics, special functions, probability theory and geometry, we refer to $[2,6,8,13,17,18]$.

\section{External ellipsoidal harmonics}

We consider the Fuchsian differential equation

$$
\prod_{j=0}^{k}\left(t-a_{j}\right)\left[v^{\prime \prime}+\sum_{j=0}^{k} \frac{\alpha_{j}+\frac{1}{2}}{t-a_{j}} v^{\prime}\right]+\left[-\frac{1}{2} \sum_{j=0}^{k} \frac{p_{j} \alpha_{j} A_{j}}{t-a_{j}}+\sum_{i=0}^{k-1} \lambda_{i} t^{i}\right] v=0
$$


for the function $v(t)$ where the prime denotes differentiation with respect to $t$, and

$$
A_{j}:=\prod_{\substack{i=0 \\ i \neq j}}^{k}\left(a_{j}-a_{i}\right)
$$

The parameters $\alpha_{j}$ are from (1.2), $\mathbf{p}=\left(p_{0}, p_{1} \ldots, p_{k}\right)$ is a fixed parity vector,

$$
a_{0}<a_{1}<\cdots<a_{k}
$$

are chosen numbers and $\lambda_{0}, \lambda_{1}, \ldots, \lambda_{k-1}$ are real spectral parameters.

Let $\mathbf{n}=\left(n_{1}, n_{2}, \ldots, n_{k}\right) \in \mathbb{N}_{0}^{k}$. In [21, Section 2] we considered the quasi-polynomial $E_{\mathbf{n}, \mathbf{p}}$ originally introduced by Stieltjes [19] in the special case $\mathbf{p}=0$. The quasi-polynomial $E_{\mathbf{n}, \mathbf{p}}$ has $n_{j}$ zeros in $\left(a_{j-1}, a_{j}\right)$ for each $j=1,2, \ldots, k$, and it is a solution of $(2.1)$ for special values of the parameters $\lambda_{0}, \lambda_{1}, \ldots, \lambda_{k-1}$. For this set of parameters, $-\frac{m}{2}$ and $\mu+\frac{m}{2}$ are the exponents of equation (2.1) at infinity, where $m$ and $\mu$ are according to (1.4), (1.5). Now $E_{\mathbf{n}, \mathbf{p}}$ is the Frobenius solution belonging to the exponent $-\frac{m}{2}$. We introduce a second Frobenius solution $\mathcal{E}_{\mathbf{n}, \mathbf{p}}$ of equation (2.1) belonging to the exponent $\mu+\frac{m}{2}$ at infinity. This solution is defined for $t>a_{k}$ and it is normalized by the condition that

$$
\lim _{t \rightarrow \infty} t^{\mu+\frac{m}{2}} \mathcal{E}_{\mathbf{n}, \mathbf{p}}(t)=1 \text {. }
$$

This normalization and the corresponding one of $E_{\mathbf{n}, \mathbf{p}}$ leads to the Wronskian

$$
\mathcal{E}_{\mathbf{n}, \mathbf{p}}(t) E_{\mathbf{n}, \mathbf{p}}^{\prime}(t)-E_{\mathbf{n}, \mathbf{p}}(t) \mathcal{E}_{\mathbf{n}, \mathbf{p}}^{\prime}(t)=(\mu+m) \prod_{j=0}^{k}\left(t-a_{j}\right)^{-\alpha_{j}-\frac{1}{2}}
$$

which holds for $t>a_{k}$. In the classical case $k=2, \boldsymbol{\alpha}=\mathbf{0}$, the second solution $\mathcal{E}_{\mathbf{n}, \mathbf{p}}$ appears in $[1, \S 9.7]$ and $[11, \S 100]$.

We introduce ellipsoidal coordinates involving the parameters $(2.2)$. For every $\left(x_{0}, \ldots, x_{k}\right)$ in the positive cone of $\mathbb{R}^{k+1}$

$$
x_{0}>0, \ldots, x_{k}>0,
$$

its ellipsoidal coordinates $t_{0}, t_{1}, \ldots, t_{k}$ lie in the intervals

$$
a_{k}<t_{0}<\infty, \quad a_{i-1}<t_{i}<a_{i}, \quad i=1, \ldots, k,
$$

and satisfy

$$
\sum_{j=0}^{k} \frac{x_{j}^{2}}{t_{i}-a_{j}}=1 \quad \text { for } \quad i=0,1, \ldots, k .
$$

These coordinates provide a bijective mapping between the positive cone (2.4) and the cube (2.5).

As explained in [21, Section 3], equation (1.1) can be solved by the method of separation of variables in ellipsoidal coordinates. In [21] we considered the internal ellipsoidal $h$-harmonic

$$
F_{\mathbf{n}, \mathbf{p}}(\mathbf{x})=\prod_{j=0}^{k} E_{\mathbf{n}, \mathbf{p}}\left(t_{j}\right)
$$

where $\left(t_{0}, t_{1}, \ldots, t_{k}\right)$ are ellipsoidal coordinates of $\mathbf{x}$. We now introduce the external ellipsoidal $h$-harmonic $\mathcal{F}_{\mathbf{n}, \mathbf{p}}$ by

$$
\mathcal{F}_{\mathbf{n}, \mathbf{p}}(\mathbf{x}):=\mathcal{E}_{\mathbf{n}, \mathbf{p}}\left(t_{0}\right) \prod_{j=1}^{k} E_{\mathbf{n}, \mathbf{p}}\left(t_{j}\right)=\frac{\mathcal{E}_{\mathbf{n}, \mathbf{p}}\left(t_{0}\right)}{E_{\mathbf{n}, \mathbf{p}}\left(t_{0}\right)} F_{\mathbf{n}, \mathbf{p}}(\mathbf{x}) .
$$


This function is originally defined in the positive cone (2.4) and can then be extended analytically to the complement of the degenerate ellipsoid

$$
\sum_{j=0}^{k-1} \frac{x_{j}^{2}}{a_{k}-a_{j}} \leq 1, \quad x_{k}=0 .
$$

We see this as follows. The ellipsoidal coordinate $t_{0}>a_{k}$ is an analytic function of $\mathbf{x}$ for $\mathbf{x}$ outside the degenerate ellipsoid (2.6). Further, $E_{\mathbf{n}, \mathbf{p}}$ and $\mathcal{E}_{\mathbf{n}, \mathbf{p}}$ are analytic functions on the interval $\left(a_{k}, \infty\right), E_{\mathbf{n}, \mathbf{p}}$ has no zero there and $F_{\mathbf{n}, \mathbf{p}}(\mathbf{x})$ is a polynomial in $\mathbf{x}$.

We note that $\mathcal{F}_{\mathbf{n}, \mathbf{p}}$ has parity $\mathbf{p}$, that is,

$$
\mathcal{F}_{\mathbf{n}, \mathbf{p}}\left(\sigma_{j}(\mathbf{x})\right)=(-1)^{p_{j}} \mathcal{F}_{\mathbf{n}, \mathbf{p}}(\mathbf{x}) .
$$

Therefore, by construction, $\mathcal{F}_{\mathbf{n}, \mathbf{p}}$ satisfies equation (1.1). It may be appropriate here to remark that a solution of (1.1) is always implicitly assumed to be defined on an open set which is invariant under the reflections $\sigma_{j}, j=0,1, \ldots, k$. Of course, our domain of definition of $\mathcal{F}_{\mathbf{n}, \mathbf{p}}$ has this property.

\section{The fundamental solution}

We introduce the function

$$
\Phi(\mathbf{x}, \mathbf{z}):=\frac{\Gamma(\mu)}{4 \prod_{j=0}^{k} \Gamma\left(\alpha_{j}+\frac{1}{2}\right)} \int_{[-1,1]^{k+1}}(\Psi(\mathbf{x}, \mathbf{z}, \boldsymbol{\tau}))^{-\mu} \prod_{j=0}^{k} c_{\alpha_{j}}\left(1+\tau_{j}\right)\left(1-\tau_{j}^{2}\right)^{\alpha_{j}-1} d \boldsymbol{\tau}
$$

where

$$
\Psi(\mathbf{x}, \mathbf{z}, \boldsymbol{\tau}):=\|\mathbf{x}\|^{2}-2\left(\tau_{0} x_{0} z_{0}+\cdots+\tau_{k} x_{k} z_{k}\right)+\|\mathbf{z}\|^{2} \geq 0
$$

$c_{\nu}:=\left(B\left(\frac{1}{2}, \nu\right)\right)^{-1}$ with $B$ denoting the Beta function, and $\mu$ is from (1.5). If $\alpha_{i}=0$ then delete the factor containing $c_{\alpha_{i}}$, set $\tau_{i}=1$ in $\Psi$ and omit the integration on $\tau_{i}$. If $\alpha_{j}=0$ for all $j$ then $\Phi$ reduces to the standard fundamental solution of Laplace's equation [10, page 22].

If $\left|x_{j}\right| \neq\left|z_{j}\right|$ for at least one $j$, then $\Psi(\mathbf{x}, \mathbf{z}, \cdot)$ has a positive lower bound on $[-1,1]^{k+1}$. Therefore, $\Phi(\mathbf{x}, \mathbf{z})$ is analytic everywhere in $\mathbb{R}^{k+1} \times \mathbb{R}^{k+1}$ except at points where $\left|x_{j}\right|=\left|z_{j}\right|$ for all $j$. We recognize that $\Phi(\mathbf{x}, \mathbf{z})=\Phi(\mathbf{z}, \mathbf{x}), \Phi(\gamma \mathbf{x}, \gamma \mathbf{z})=|\gamma|^{-2 \mu} \Phi(\mathbf{x}, \mathbf{z})$ and $\Phi(\mathbf{x}, \mathbf{z})>0$. Moreover,

$$
\Phi(\mathbf{0}, \mathbf{z})=\frac{\Gamma(\mu)}{4 \prod_{j=0}^{k} \Gamma\left(\alpha_{j}+\frac{1}{2}\right)}\|\mathbf{z}\|^{-2 \mu}
$$

If $\mathbf{z} \neq \mathbf{0}$ we can expand $\Phi(\cdot, \mathbf{z})$ in a Taylor series at $\mathbf{x}=0$. Its coefficients are given by the following lemma.

Lemma 1. If $\mathbf{z} \neq \mathbf{0}$ then, for every $\left(q_{0}, q_{1}, \ldots, q_{k}\right) \in \mathbb{N}_{0}^{k+1}$,

$$
\begin{aligned}
& \left.\mathcal{D}_{x_{0}}^{q_{0}} \cdots \mathcal{D}_{x_{k}}^{q_{k}}\left(x_{0}^{q_{0}} \cdots x_{k}^{q_{k}}\right) \frac{\partial_{x_{0}}^{q_{0}} \cdots \partial_{x_{k}}^{q_{k}} \Phi(\mathbf{x}, \mathbf{z})}{q_{0} ! \cdots q_{k} !}\right|_{\mathbf{x}=\mathbf{0}} \\
& =\left.\mathcal{D}_{x_{0}}^{q_{0}} \cdots \mathcal{D}_{x_{k}}^{q_{k}} \Phi(\mathbf{x}, \mathbf{z})\right|_{\mathbf{x}=\mathbf{0}}=(-1)^{|\mathbf{q}|} \mathcal{D}_{z_{0}}^{q_{0}} \cdots \mathcal{D}_{z_{k}}^{q_{k}} \Phi(\mathbf{0}, \mathbf{z}),
\end{aligned}
$$

where $|\mathbf{q}|=q_{0}+\cdots+q_{k}$. 
Proof. We note that the first equality in (3.2) is trivial so it is enough to prove the second one. The proof of the second equality involves some lengthy formulas, so we will use some common abbreviations. If $\mathbf{j}=\left(j_{0}, j_{1}, \ldots, j_{k}\right)$ is a multi-index we set $\mathbf{j} !=j_{0} ! \cdots j_{k} !, \partial_{\mathbf{x}}^{\mathbf{j}}=\partial_{x_{0}}^{j_{0}} \cdots \partial_{x_{k}}^{j_{k}}$ and $\mathbf{x}^{\mathbf{j}}=x_{0}^{j_{0}} \cdots x_{k}^{j_{k}}$. By Faa di Bruno's formula (or by equation [21, (5.4)]), we write

$$
\partial_{\mathbf{x}}^{\mathbf{q}} f(\Psi(\mathbf{x}, \mathbf{z}, \boldsymbol{\tau}))=\sum_{\mathbf{j}} 2^{|\mathbf{q}|-2|\mathbf{j}|} f^{(|\mathbf{q}|-|\mathbf{j}|)}(\Psi(\mathbf{x}, \mathbf{z}, \boldsymbol{\tau})) \frac{1}{\mathbf{j} !} \partial_{\mathbf{x}}^{2 \mathbf{j}}\left(x_{0}-\tau_{0} z_{0}\right)^{q_{0}} \cdots\left(x_{k}-\tau_{k} z_{k}\right)^{q_{k}},
$$

where $f(u):=u^{-\mu}$, and the summation is over all multi-indices $\mathbf{j}=\left(j_{0}, j_{1}, \ldots, j_{k}\right)$ with $j_{i} \geq 0$ and $q_{i}-2 j_{i} \geq 0, i=0,1, \ldots, k$. Computing the partial derivatives on the right-hand side and setting $\mathbf{x}=\mathbf{0}$ gives

$$
\begin{aligned}
\partial_{\mathbf{x}}^{\mathbf{q}} f & \left.(\Psi(\mathbf{x}, \mathbf{z}, \boldsymbol{\tau}))\right|_{\mathbf{x}=\mathbf{0}} \\
& =(-1)^{|\mathbf{q}|} \sum_{\mathbf{j}} 2^{|\mathbf{q}|-2|\mathbf{j}|} f^{(|\mathbf{q}|-|\mathbf{j}|)}\left(\|\mathbf{z}\|^{2}\right) \frac{\mathbf{q} !}{\mathbf{j} !(\mathbf{q}-2 \mathbf{j}) !}\left(\tau_{0} z_{0}\right)^{q_{0}-2 j_{0}} \cdots\left(\tau_{k} z_{k}\right)^{q_{k}-2 j_{k}} .
\end{aligned}
$$

We now carry out the integrations indicated in (3.1) taking into account that

$$
c_{\alpha_{j}} \int_{-1}^{1} \tau_{j}^{m}\left(1+\tau_{j}\right)\left(1-\tau_{j}^{2}\right)^{\alpha_{j}-1} d \tau_{j}=\frac{m !}{\mathcal{D}_{z_{j}}^{m}\left(z_{j}^{m}\right)} .
$$

Note that $\mathcal{D}_{z_{j}}^{m}\left(z_{j}^{m}\right)$ is a constant depending on $j$ and $m$ (the "Dunkl factorial".) We obtain

$$
\left.\partial_{\mathbf{x}}^{\mathbf{q}} \tilde{\Phi}(\mathbf{x}, \mathbf{z})\right|_{\mathbf{x}=\mathbf{0}}=(-1)^{|\mathbf{q}|} \sum_{\mathbf{j}} 2^{|\mathbf{q}|-2|\mathbf{j}|} f^{(|\mathbf{q}|-|\mathbf{j}|)}\left(\|\mathbf{z}\|^{2}\right) \frac{\mathbf{q} !}{\mathbf{j} ! \mathcal{D}_{\mathbf{z}}^{\mathbf{q}-2 \mathbf{j}} \mathbf{z}^{\mathbf{q}-2 \mathbf{j}}} \mathbf{z}^{\mathbf{q}-2 \mathbf{j}}
$$

where $\tilde{\Phi}$ is defined by the right-hand side of (3.1) but with the normalization factor in front of the integral omitted. Now we use the first identity in (3.2) to obtain

$$
\begin{aligned}
\left.\mathcal{D}_{\mathbf{x}}^{\mathbf{q}} \tilde{\Phi}(\mathbf{x}, \mathbf{z})\right|_{\mathbf{x}=\mathbf{0}} & =(-1)^{|\mathbf{q}|} \sum_{\mathbf{j}} 2^{|\mathbf{q}|-2|\mathbf{j}|} f^{(|\mathbf{q}|-|\mathbf{j}|)}\left(\|\mathbf{z}\|^{2}\right) \frac{1}{\mathbf{j} !} \frac{\mathcal{D}_{\mathbf{z}}^{\mathbf{q}} \mathbf{z}^{\mathbf{q}}}{\mathcal{D}_{\mathbf{z}}^{\mathbf{q}-2 \mathbf{j}} \mathbf{z}^{\mathbf{q}-2 \mathbf{j}}} \mathbf{z}^{\mathbf{q}-2 \mathbf{j}} \\
& =(-1)^{|\mathbf{q}|} \sum_{\mathbf{j}} 2^{|\mathbf{q}|-2|\mathbf{j}|} f^{(|\mathbf{q}|-|\mathbf{j}|)}\left(\|\mathbf{z}\|^{2}\right) \frac{1}{\mathbf{j} !} \mathcal{D}_{\mathbf{z}}^{2 \mathbf{j}} \mathbf{z}^{\mathbf{q}}
\end{aligned}
$$

Finally, we apply [21, Lemma 2] to the function $A\left(v_{0}, \ldots, v_{k}\right)=f\left(v_{0}+\cdots+v_{k}\right)$ and obtain the second identity in (3.2).

Since $\|\mathbf{z}\|^{-2 \mu}$ is $h$-harmonic, Lemma 1 shows that $\Phi(\mathbf{x}, \cdot)$ is $h$-harmonic for fixed $\mathbf{x}$ and so, by symmetry, $\Phi(\cdot, \mathbf{z})$ is $h$-harmonic for fixed $\mathbf{z}$.

Knowledge of the exact nature of the singularities of $\Phi$ will be immaterial in this paper. An analysis of the singularities would be along the following lines. We see that the singularity of $\Phi(\mathbf{x}, \mathbf{z})$ at $\mathbf{x}=\mathbf{z}$ is determined by integration over values $\tau_{j}$ close to 1 so it is convenient to substitute $\sigma_{j}=1-\tau_{j}$. If all $z_{j}$ are nonzero we have the elementary inequality

$$
\Psi(\mathbf{x}, \mathbf{z}, \boldsymbol{\tau}) \geq c\left(\|\mathbf{x}-\mathbf{z}\|^{2}+\|\boldsymbol{\sigma}\|\right)
$$

if $\mathbf{x}$ is close enough to $\mathbf{z}$ and $c$ is a positive constant; see the proof of [9, Theorem 5.5.7] for similar estimates. It follows that $\Phi(\cdot, \mathbf{z})$ and its normal derivative on spheres $\|\mathbf{x}-\mathbf{z}\|=\epsilon$ do not grow faster as $\epsilon \rightarrow 0$ than in the classical case $\boldsymbol{\alpha}=\mathbf{0}$. Obvious modifications of the growth rate are obtained when one or several $z_{j}$ vanish. If $\left|x_{j}\right|=\left|z_{j}\right|$ for all $j$ but $x_{i} \neq z_{i}$ for at least one $i$ 
then the factor $1+\tau_{i}$ appearing in (3.1) comes into play and makes the singularity milder than that at $\mathbf{x}=\mathbf{z}$.

We now express $\Phi$ in terms of the reproducing kernel $P_{m}(\mathbf{x}, \mathbf{y})$ of the linear space of spherical $h$-harmonics of degree $m$ with respect to the inner product (1.3); see [9, Section 5.3]. We normalize the reproducing kernel so that

$$
Y(\mathbf{x})=\int_{\mathbf{S}^{k}} h^{2}(\mathbf{x}) P_{m}(\mathbf{x}, \mathbf{y}) Y(\mathbf{y}) d \mathbf{S}(\mathbf{y})
$$

for every spherical $h$-harmonic $Y$ of degree $m$ and every $\mathbf{x} \in \mathbf{S}^{k}$. The generating function for Gegenbauer polynomials [9, Definition 1.4.10] is given by

$$
\left(1-2 u s+s^{2}\right)^{-\mu}=\sum_{m=0}^{\infty} C_{m}^{\mu}(u) s^{m} .
$$

The convergence is uniform for $-1 \leq u \leq 1,|s| \leq q<1$. In (3.3) we set $s=\|\mathbf{x}\| /\|\mathbf{z}\|$ and $u=\|\mathbf{x}\|^{-1}\|\mathbf{z}\|^{-1}\left(\tau_{0} x_{0} z_{0}+\cdots+\tau_{k} x_{k} z_{k}\right)$. Then (3.1) and the integral representation of the reproducing kernel [9, Theorem 5.5.5] yields the following theorem.

Theorem 1. If $\|\mathbf{x}\|<\|\mathbf{z}\|$ then

$$
\Phi(\mathbf{x}, \mathbf{z})=\sum_{m=0}^{\infty} \frac{1}{2(\mu+m)}\|\mathbf{x}\|^{m}\|\mathbf{z}\|^{-2 \mu-m} P_{m}\left(\frac{\mathbf{x}}{\|\mathbf{x}\|}, \frac{\mathbf{z}}{\|\mathbf{z}\|}\right) .
$$

The convergence is uniform for $\frac{\|\mathbf{x}\|}{\|\mathbf{z}\|} \leq q<1$.

When $k=2, \boldsymbol{\alpha}=\mathbf{0}$, formula (3.4) shows how to expand the reciprocal distance between $\mathbf{z}$ and $\mathbf{x}$ into a series of Legendre polynomials. This expansion is due to Laplace and it marks the very beginning of the history of spherical harmonics [11, page 3].

\section{Integral formulas for external $h$-harmonics}

Multiplying (3.4) by a spherical $h$-harmonic and integrating, we obtain an integral representation of external spherical $h$-harmonics.

Theorem 2. Let $Y$ be a spherical h-harmonic of degree $m$, and let $\mathcal{Y}$ denote the corresponding external spherical h-harmonic $\mathcal{Y}(\mathbf{x}):=\|\mathbf{x}\|^{-2 \mu-2 m} Y(\mathbf{x})$. Then, for $\|\mathbf{z}\|>1$,

$$
\mathcal{Y}(\mathbf{z})=2(\mu+m) \int_{\mathbf{S}^{k}} h^{2}(\mathbf{x}) \Phi(\mathbf{x}, \mathbf{z}) Y(\mathbf{x}) d \mathbf{S}(\mathbf{x}) .
$$

In particular, Theorem 2 can be applied to a sphero-conal $h$-harmonic $Y=G_{\mathbf{n}, \mathbf{p}}$.

Let $Y$ be any spherical $h$-harmonic of degree $m$. If $\|\mathbf{z}\|>1$ we use Green's formula for $h$-harmonic functions [9, Lemma 5.1.5] inside the unit ball to obtain

$$
0=\int_{\mathbf{S}^{k}} h^{2}(\mathbf{x})\left(Y(\mathbf{x}) \frac{\partial \Phi(\mathbf{x}, \mathbf{z})}{\partial \nu_{\mathbf{x}}}-\Phi(\mathbf{x}, \mathbf{z}) \frac{\partial Y(\mathbf{x})}{\partial \nu}\right) d \mathbf{S}(\mathbf{x})
$$

where $\nu$ denotes the outward normal. On the unit sphere we have

$$
\frac{\partial Y}{\partial \nu}=m Y=m \mathcal{Y}, \quad \frac{\partial \mathcal{Y}}{\partial \nu}=(-2 \mu-m) Y .
$$


Therefore, adding (4.2) to (4.1) gives

$$
\mathcal{Y}(\mathbf{z})=\int_{\mathbf{S}^{k}} h^{2}(\mathbf{x})\left(\mathcal{Y}(\mathbf{x}) \frac{\partial \Phi(\mathbf{x}, \mathbf{z})}{\partial \nu_{\mathbf{x}}}-\Phi(\mathbf{x}, \mathbf{z}) \frac{\partial \mathcal{Y}(\mathbf{x})}{\partial \nu}\right) d \mathbf{S}(\mathbf{x}) .
$$

When $\boldsymbol{\alpha}=0$ this formula corresponds exactly to the well-known representation formula of harmonic functions in terms of a fundamental solution [10, page 34]. By using Green's formula in the region between two spheres we see that we also have

$$
\mathcal{Y}(\mathbf{z})=\int_{\mathbf{S}_{R}^{k}} h^{2}(\mathbf{x})\left(\mathcal{Y}(\mathbf{x}) \frac{\partial \Phi(\mathbf{x}, \mathbf{z})}{\partial \nu_{\mathbf{x}}}-\Phi(\mathbf{x}, \mathbf{z}) \frac{\partial \mathcal{Y}(\mathbf{x})}{\partial \nu}\right) d \mathbf{S}(\mathbf{x})
$$

when $\|\mathbf{z}\|>R$, where

$$
\mathbf{S}_{R}^{k}:=\{\mathbf{x}:\|\mathbf{x}\|=R\} .
$$

We introduce ellipsoidal coordinates by choosing numbers $a_{j}$ according to (2.2), and prove an integral representation of external ellipsoidal $h$-harmonics.

Theorem 3. Let $\mathbf{J}$ denote the ellipsoid

$$
\mathbf{J}=\left\{\mathbf{y}: \sum_{j=0}^{k} \frac{y_{j}^{2}}{t-a_{j}}=1\right\}
$$

for some fixed $t>a_{k}$. Then, for all $\mathbf{z}$ exterior to $\mathbf{J}$,

$$
\mathcal{F}_{\mathbf{n}, \mathbf{p}}(\mathbf{z})=\frac{2(\mu+m)}{E_{\mathbf{n}, \mathbf{p}}^{2}(t)} \prod_{j=0}^{k}\left(t-a_{j}\right)^{-\alpha_{j}-\frac{1}{2}} \int_{\mathbf{J}} h^{2}(\mathbf{y}) w(\mathbf{y}) \Phi(\mathbf{y}, \mathbf{z}) F_{\mathbf{n}, \mathbf{p}}(\mathbf{y}) d \mathbf{J}(\mathbf{y}),
$$

where $m=2|\mathbf{n}|+|\mathbf{p}|$ and $w$ is defined by

$$
\frac{1}{w(\mathbf{y})}:=\sum_{j=0}^{k} \frac{y_{j}^{2}}{\left(t-a_{j}\right)^{2}} \text {. }
$$

Proof. We will suppress the subscripts $\mathbf{n}, \mathbf{p}$ in this proof. Both sides of equation (4.5) are analytic in the exterior of $\mathbf{J}$ so it will be sufficient to prove the equation for $\|\mathbf{z}\|>R$, where $R$ is chosen so large that $\mathbf{J}$ fits inside the sphere $\mathbf{S}_{R}^{k}$.

We claim that formula (4.3) is also true if we replace $\mathcal{Y}$ by $\mathcal{F}$. To see this we expand $\mathcal{F}$ on the sphere $\mathbf{S}_{R}^{k}$ in a series

$$
\mathcal{F}(\mathbf{x})=\sum_{q=0}^{\infty} Y_{q}(\mathbf{x})
$$

where $Y_{q}$ is a spherical $h$-harmonic of degree $q$. Using an uniqueness theorem for $h$-harmonic functions defined in the exterior of spheres with proper decay at infinity (the analogue of [7, Theorem 2.9]) we obtain

$$
\mathcal{F}(\mathbf{x})=\sum_{q=0}^{\infty} R^{2 \mu+2 q}\|\mathbf{x}\|^{-2 \mu-2 q} Y_{q}(\mathbf{x}) .
$$

This series and the series obtained by term-by-term differentiation converge uniformly to the proper limits on and exterior to $\mathbf{S}_{R}^{k}$. Therefore, by taking limits we obtain

$$
\mathcal{F}(\mathbf{z})=\int_{\mathbf{S}_{R}^{k}} h^{2}(\mathbf{x})\left(\mathcal{F}(\mathbf{x}) \frac{\partial \Phi(\mathbf{x}, \mathbf{z})}{\partial \nu_{\mathbf{x}}}-\Phi(\mathbf{x}, \mathbf{z}) \frac{\partial \mathcal{F}(\mathbf{x})}{\partial \nu}\right) d \mathbf{S}(\mathbf{x}) .
$$


Using Green's formula in the region between $\mathbf{J}$ and $\mathbf{S}_{R}^{k}$, we also have

$$
\mathcal{F}(\mathbf{z})=\int_{\mathbf{J}} h^{2}(\mathbf{y})\left(\mathcal{F}(\mathbf{y}) \frac{\partial \Phi(\mathbf{y}, \mathbf{z})}{\partial \nu_{\mathbf{y}}}-\Phi(\mathbf{y}, \mathbf{z}) \frac{\partial \mathcal{F}(\mathbf{y})}{\partial \nu}\right) d \mathbf{J}(\mathbf{y})
$$

where $\nu$ denotes the outward unit normal vector. We now apply Green's formula to the internal ellipsoidal $h$-harmonic $F$ in the interior of $\mathbf{J}$ and obtain

$$
\int_{\mathbf{J}} h^{2}(\mathbf{y})\left(F(\mathbf{y}) \frac{\partial \Phi(\mathbf{y}, \mathbf{z})}{\partial \nu_{\mathbf{y}}}-\Phi(\mathbf{y}, \mathbf{z}) \frac{\partial F(\mathbf{y})}{\partial \nu}\right) d \mathbf{J}(\mathbf{y})=0 .
$$

We multiply (4.7) by $E(t),(4.8)$ by $\mathcal{E}(t)$ and subtract noting that $E(t) \mathcal{F}(\mathbf{y})=\mathcal{E}(t) F(\mathbf{y})$ on $\mathbf{J}$. We obtain

$$
E(t) \mathcal{F}(\mathbf{z})=\int_{\mathbf{J}} h^{2}(\mathbf{y}) \Phi(\mathbf{y}, \mathbf{z})\left(\mathcal{E}(t) \frac{\partial F(\mathbf{y})}{\partial \nu}-E(t) \frac{\partial \mathcal{F}(\mathbf{y})}{\partial \nu}\right) d \mathbf{J}(\mathbf{y})
$$

Since the ellipsoidal coordinates are orthogonal, the normal derivative is expressible in terms of the derivative with respect to the ellipsoidal coordinate $t_{0}$ :

$$
\frac{\partial}{\partial \nu}=2 w(\mathbf{y}) \frac{\partial}{\partial t_{0}}
$$

Therefore, we find

$$
\mathcal{F}(\mathbf{z})=2 \frac{\mathcal{E}(t) E^{\prime}(t)-E(t) \mathcal{E}^{\prime}(t)}{E^{2}(t)} \int_{\mathbf{J}} h^{2}(\mathbf{y}) w(\mathbf{y}) \Phi(\mathbf{y}, \mathbf{z}) F(\mathbf{y}) d \mathbf{J}(\mathbf{y}) .
$$

We replace the Wronskian by (2.3) and (4.5) is established.

In Section 6 the integral in (4.5) will be evaluated using the formulas given in Section 5 .

Theorem 3 leads to an expansion of $\Phi$ in ellipsoidal $h$-harmonics.

Theorem 4. Let the ellipsoidal $t_{0}$-coordinate of $\mathbf{z}$ be larger than the corresponding one for $\mathbf{y}$. Then

$$
\Phi(\mathbf{y}, \mathbf{z})=\sum_{\mathbf{n}, \mathbf{p}} \frac{e_{\mathbf{n}, \mathbf{p}}^{2}}{2(\mu+2|\mathbf{n}|+|\mathbf{p}|)} F_{\mathbf{n}, \mathbf{p}}(\mathbf{y}) \mathcal{F}_{\mathbf{n}, \mathbf{p}}(\mathbf{z}),
$$

where the positive constants $e_{\mathbf{n}, \mathbf{p}}$ are determined by

$$
e_{\mathbf{n}, \mathbf{p}}^{2} \int_{\mathbf{S}^{k}} h^{2}(\mathbf{x}) G_{\mathbf{n}, \mathbf{p}}^{2}(\mathbf{x}) d \mathbf{S}(\mathbf{x})=1
$$

Proof. Let $\mathbf{J}$ be the ellipsoid (4.4) that contains $\mathbf{y}$. The function $\Phi(\cdot, \mathbf{z})$ is analytic and $h$ harmonic on and inside $\mathbf{J}$. Therefore, it can be expanded in ellipsoidal $h$-harmonics as in [21, Section 7] and the expansion coefficients can be evaluated using (4.5). This gives (4.9) after a simple calculation.

When $k=2, \boldsymbol{\alpha}=\mathbf{0}$, formula (4.9) can be found in [12, page 172]. 


\section{Integral formulas for spherical $h$-harmonics}

Theorem 5. Let $f$ be a homogeneous polynomial of degree $\ell$, and let $Y$ be a spherical h-harmonic of degree $m$. If $\ell=m+2 r, r=0,1,2, \ldots$, then

$$
\int_{\mathbf{S}^{k}} h^{2}(\mathbf{x}) f(\mathbf{x}) Y(\mathbf{x}) d \mathbf{S}(\mathbf{x})=\frac{1}{2^{\ell-1} r !} \frac{\prod_{i=0}^{k} \Gamma\left(\alpha_{i}+\frac{1}{2}\right)}{\Gamma(m+\mu+r+1)} \Delta_{h}^{r} Y(\mathcal{D}) f(\mathbf{x}),
$$

where $Y(\mathcal{D})=Y\left(\mathcal{D}_{0}, \ldots, \mathcal{D}_{k}\right)$. If $\ell<m$ or $\ell-m$ is odd then the integral in (5.1) vanishes.

Proof. Theorem 5.1.15 in [9] gives the expansion of $f$ in spherical $h$-harmonics on $\mathbf{S}_{k}$. It follows from this expansion and [9, Theorem 5.1.6] that the integral in (5.1) vanishes when $\ell<m$ or when $\ell-m$ is odd. If $\ell=m+2 r, r=0,1,2, \ldots$, then [9, Theorem 5.1.15] shows that

$$
\int_{\mathbf{S}^{k}} h^{2}(\mathbf{x}) f(\mathbf{x}) Y(\mathbf{x}) d \mathbf{S}(\mathbf{x})=\int_{\mathbf{S}^{k}} h^{2}(\mathbf{x}) \tilde{f}(\mathbf{x}) Y(\mathbf{x}) d \mathbf{S}(\mathbf{x}),
$$

where $\tilde{f}$ is a homogeneous polynomial of degree $m$ given by

$$
\tilde{f}(\mathbf{x})=\frac{1}{4^{r} r !(m+\mu+1)_{r}} \Delta_{h}^{r} f(\mathbf{x})
$$

We evaluate the integral on the right-hand side of (5.2) by applying Theorem 5.2.4 in [9]. We obtain

$$
\int_{\mathbf{S}^{k}} h^{2}(\mathbf{x}) f(\mathbf{x}) Y(\mathbf{x}) d \mathbf{S}(\mathbf{x})=\frac{1}{c^{\prime} 2^{m} 4^{r} r !(\mu+1)_{m}(m+\mu+1)_{r}} \Delta_{h}^{r} Y(\mathcal{D}) f(\mathbf{x}),
$$

where

$$
\frac{1}{c^{\prime}}=\int_{\mathbf{S}^{k}} h^{2}(\mathbf{x}) d \mathbf{S}(x)=\frac{2 \prod_{i=0}^{k} \Gamma\left(\alpha_{i}+\frac{1}{2}\right)}{\Gamma(\mu+1)} .
$$

After simplification of the constant factors we arrive at the desired equation (5.1).

In the special case $k=2, \boldsymbol{\alpha}=\mathbf{0}$ formula (5.1) is due to Hobson [14, $\S 100]$.

We state a consequence of Theorem 1 .

Corollary 1. Let $f(\mathbf{x})=\sum_{\ell=0}^{\infty} f_{\ell}(\mathbf{x})$, where $f_{\ell}$ is a homogeneous polynomial of degree $\ell$, and the convergence is uniform on $\mathbf{S}^{k}$. Let $Y$ be a spherical h-harmonic of degree $m$. Then

$$
\int_{\mathbf{S}^{k}} h^{2}(\mathbf{x}) f(\mathbf{x}) Y(\mathbf{x}) d \mathbf{S}(\mathbf{x})=\sum_{r=0}^{\infty} \frac{1}{2^{m+2 r-1} r !} \frac{\prod_{i=0}^{k} \Gamma\left(\alpha_{i}+\frac{1}{2}\right)}{\Gamma(m+\mu+r+1)} \Delta_{h}^{r} Y(\mathcal{D}) f_{m+2 r}(\mathbf{x}) .
$$

\section{Niven's formula for external ellipsoidal $h$-harmonics}

We consider the ellipsoid $\mathbf{J}$ from (4.4) with semi-axes $d_{j}:=\sqrt{t-a_{j}}$. By substituting $y_{j}=d_{j} x_{j}$, $\mathbf{y} \in \mathbf{J}$ is transformed to $\mathbf{x} \in \mathbf{S}^{k}$. If $f(\mathbf{x})$ is a continuous function on $\mathbf{S}^{k}$, then

$$
g(\mathbf{y}):=f\left(\frac{y_{0}}{d_{0}}, \ldots, \frac{y_{k}}{d_{k}}\right)
$$


becomes a continuous function on $\mathbf{J}$ and

$$
\int_{\mathbf{S}^{k}} f(\mathbf{x}) d \mathbf{S}(\mathbf{x})=\prod_{j=0}^{k} d_{j}^{-1} \int_{\mathbf{J}} w(\mathbf{y}) g(\mathbf{y}) d \mathbf{J}(\mathbf{y})
$$

with $w(\mathbf{y})$ from (4.6).

Let $Y$ denote a spherical $h$-harmonic of degree $m$, and let $f$ be as in Corollary 1 . We use the substitution $y_{j}=d_{j} x_{j}$ and (6.2) to write (5.3) as

$$
\begin{aligned}
\int_{\mathbf{J}} h^{2}(\mathbf{y}) w(\mathbf{y}) g(\mathbf{y}) Y\left(\frac{y_{0}}{d_{0}}, \ldots, \frac{y_{k}}{d_{k}}\right) d \mathbf{J}(\mathbf{y}) \\
=\prod_{j=0}^{k} d_{j}^{2 \alpha_{j}+1} \sum_{r=0}^{\infty} \frac{1}{2^{m+2 r-1} r !} \frac{\prod_{j=0}^{k} \Gamma\left(\alpha_{j}+\frac{1}{2}\right)}{\Gamma(m+\mu+r+1)} \Lambda^{r} Y\left(d_{0} \mathcal{D}_{0}, \ldots, d_{k} \mathcal{D}_{k}\right) g_{m+2 r}(\mathbf{x}),
\end{aligned}
$$

where $\Lambda$ is the operator

$$
\Lambda:=d_{0}^{2} \mathcal{D}_{0}^{2}+\cdots+d_{k}^{2} \mathcal{D}_{k}^{2}
$$

and $g$ is defined by (6.1) with the same connection between $f_{\ell}$ and $g_{\ell}$. We use (6.3) in the special case $Y=G_{\mathbf{n}, \mathbf{p}}, m=2|\mathbf{n}|+|\mathbf{p}|$, and $g=\Phi(\cdot, \mathbf{z})$, where $\|\mathbf{z}\|>d_{0}$ (the largest semi-axis of $\mathbf{J})$. We also note that

$$
E_{\mathbf{n}, \mathbf{p}}(t) G_{\mathbf{n}, \mathbf{p}}\left(\frac{y_{0}}{d_{0}}, \ldots, \frac{y_{k}}{d_{k}}\right)=F_{\mathbf{n}, \mathbf{p}}(\mathbf{y})
$$

see $[21,(6.1)]$. Then the integral representation (4.5) for the external ellipsoidal $h$-harmonic $\mathcal{F}_{\mathbf{n}, \mathbf{p}}$ and (6.3) yield

$$
\begin{aligned}
\mathcal{F}_{\mathbf{n}, \mathbf{p}}(\mathbf{z})= & \frac{2(\mu+m)}{E_{\mathbf{n}, \mathbf{p}}(t)} \sum_{r=0}^{\infty} \frac{1}{2^{m+2 r-1} r !} \frac{\prod_{j=0}^{k} \Gamma\left(\alpha_{j}+\frac{1}{2}\right)}{\Gamma(m+\mu+r+1)} \\
& \times \Lambda^{r} G_{\mathbf{n}, \mathbf{p}}\left(d_{0} \mathcal{D}_{x_{0}}, \ldots, d_{k} \mathcal{D}_{x_{k}}\right) \Phi_{m+2 r}(\mathbf{x}, \mathbf{z}) .
\end{aligned}
$$

We now use the identity

$$
G_{\mathbf{n}, \mathbf{p}}\left(d_{0} \mathcal{D}_{0}, \ldots, d_{k} \mathcal{D}_{k}\right) g(\mathbf{x})=E_{\mathbf{n}, \mathbf{p}}(t) G_{\mathbf{n}, \mathbf{p}}(\mathcal{D}) g(\mathbf{x})
$$

which holds for any $h$-harmonic function $g$; see the formula before (6.3) in [21]. Then we replace the operator $-\Lambda$ by $a_{0} \mathcal{D}_{0}^{2}+\cdots+a_{k} \mathcal{D}_{k}^{2}$ which is possible because $\Phi(\cdot, \mathbf{z})$ is $h$-harmonic. Finally, we use Lemma 1 and arrive at the following theorem.

Theorem 6. The external ellipsoidal h-harmonic $\mathcal{F}_{\mathbf{n}, \mathbf{p}}$ admits the differential-difference representation

$$
\mathcal{F}_{\mathbf{n}, \mathbf{p}}(\mathbf{z})=\sum_{r=0}^{\infty} \frac{(-1)^{r+m}(\mu+m)}{2^{m+2 r} r !(\mu)_{m+r+1}}\left(a_{0} \mathcal{D}_{0}^{2}+\cdots+a_{k} \mathcal{D}_{k}^{2}\right)^{r} G_{\mathbf{n}, \mathbf{p}}(\mathcal{D})\|\mathbf{z}\|^{-2 \mu},
$$

where $m=2|\mathbf{n}|+|\mathbf{p}|$ and $\mu$ is defined in (1.5). The expansion is valid for $\|\mathbf{z}\|^{2}>a_{k}-a_{0}$. 
Formula (6.4) is true for all $\mathbf{z}$ with $\|\mathbf{z}\|^{2}>a_{k}-a_{0}$ because we can choose the number $t$ defining the ellipsoid (4.4) very close to $a_{k}$.

We can rewrite (6.4) slightly by using the connection between internal and external spherical $h$-harmonics [21, Corollary 2]:

$$
\mathcal{Y}(\mathbf{x})=\frac{(-1)^{m}}{2^{m}(\mu)_{m}} Y(\mathcal{D})\|\mathbf{x}\|^{-2 \mu} .
$$

This leads to the following corollary.

Corollary 2. The external ellipsoidal h-harmonic $\mathcal{F}_{\mathbf{n}, \mathbf{p}}$ admits the differential-difference representation

$$
\mathcal{F}_{\mathbf{n}, \mathbf{p}}(\mathbf{z})=\sum_{r=0}^{\infty} \frac{(-1)^{r}}{2^{2 r} r !(\mu+m+1)_{r}}\left(a_{0} \mathcal{D}_{0}^{2}+\cdots+a_{k} \mathcal{D}_{k}^{2}\right)^{r} \mathcal{G}_{\mathbf{n}, \mathbf{p}}(\mathbf{z})
$$

for $\|\mathbf{z}\|^{2}>a_{k}-a_{0}$.

\section{$7 \quad$ Examples}

We illustrate some results of this paper in the planar case $k=1$. We choose $a_{0}=-1, a_{1}=1$ and set $\alpha:=\alpha_{1}-\frac{1}{2}, \beta:=\alpha_{0}-\frac{1}{2}$. If $p_{0}=p_{1}=0$ equation (2.1) becomes

$$
\left(t^{2}-1\right)\left(v^{\prime \prime}+\frac{\alpha+1}{t-1} v^{\prime}+\frac{\beta+1}{t+1} v^{\prime}\right)+\lambda_{0} v=0 .
$$

This equation is satisfied by the Jacobi polynomial $v(t)=P_{n}^{(\alpha, \beta)}(t)$ when $\lambda_{0}=-n(n+\alpha+\beta+1)$. Therefore, the Stieltjes polynomial $E_{n, 0}$ is a multiple of $P_{n}^{(\alpha, \beta)}$. In [21] the Stieltjes polynomials were normalized such that their leading coefficient equals 1 . This gives

$$
E_{n, \mathbf{0}}=a_{n} P_{n}^{(\alpha, \beta)},
$$

where

$$
a_{n}=a_{n}^{(\alpha, \beta)}:=\frac{n ! 2^{n}}{(\alpha+\beta+n+1)_{n}} .
$$

There are similar formulas for the other three parity vectors $\mathbf{p}$; compare [21, $\S 2]$.

A second solution of (7.1) with $\lambda_{0}=-n(n+\alpha+\beta+1)$ is $Q_{n}^{(\alpha, \beta)}$; see [20, $\left.\S 4.61\right]$. Comparing the behavior at infinity, we see that

$$
\mathcal{E}_{n, \mathbf{0}}=b_{n} Q_{n}^{(\alpha, \beta)},
$$

where

$$
b_{n}=b_{n}^{(\alpha, \beta)}:=2^{-n-\alpha-\beta} \frac{\Gamma(2 n+\alpha+\beta+2)}{\Gamma(n+\alpha+1) \Gamma(n+\beta+1)} .
$$

Again, similar formulas hold for the other three parity vectors $\mathbf{p}$.

The planar sphero-conal coordinates $r, s_{1}$ as defined in [21, $\left.\S 4\right]$ essentially agree with polar coordinates $x_{0}=r \cos \phi, x_{1}=r \sin \phi$. The connection is given by $\cos (2 \phi)=s_{1}$. The internal sphero-conal $h$-harmonic $G_{n, \mathbf{0}}$ is equal to $r^{2 n} E_{n, \mathbf{0}}\left(s_{1}\right)$ in sphero-conal coordinates. Therefore, in cartesian coordinates, we obtain

$$
G_{n, \mathbf{0}}\left(x_{0}, x_{1}\right)=a_{n}\left(x_{0}^{2}+x_{1}^{2}\right)^{n} P_{n}^{(\alpha, \beta)}\left(\frac{x_{0}^{2}-x_{1}^{2}}{x_{0}^{2}+x_{1}^{2}}\right) .
$$


The external sphero-conal $h$-harmonic $\mathcal{G}_{n, \mathbf{0}}$ is given by

$$
\mathcal{G}_{n, \mathbf{0}}\left(x_{0}, x_{1}\right)=a_{n}\left(x_{0}^{2}+x_{1}^{2}\right)^{-n-\alpha-\beta-1} P_{n}^{(\alpha, \beta)}\left(\frac{x_{0}^{2}-x_{1}^{2}}{x_{0}^{2}+x_{1}^{2}}\right) .
$$

The internal ellipsoidal $h$-harmonic $F_{n, \mathbf{0}}$ takes the form $E_{n, \mathbf{0}}\left(t_{0}\right) E_{n, \mathbf{0}}\left(t_{1}\right)$ in ellipsoidal coordinates. We can express $t_{0}, t_{1}$ explicitly as functions of $x_{0}, x_{1}$ as follows

$$
\begin{aligned}
& t_{0}=\frac{1}{2}\left(x_{0}^{2}+x_{1}^{2}\right)^{2}+\left(\frac{1}{4}\left(x_{0}^{2}+x_{1}^{2}\right)^{2}-x_{0}^{2}+x_{1}^{2}+1\right)^{1 / 2}, \\
& t_{1}=\frac{1}{2}\left(x_{0}^{2}+x_{1}^{2}\right)^{2}-\left(\frac{1}{4}\left(x_{0}^{2}+x_{1}^{2}\right)^{2}-x_{0}^{2}+x_{1}^{2}+1\right)^{1 / 2} .
\end{aligned}
$$

It should be mentioned that analogous formulas do not exist when $k \geq 4$ and would be very complicated when $k=2$ or $k=3$. Now internal ellipsoidal $h$-harmonics can be written as

$$
F_{n, \mathbf{0}}\left(x_{0}, x_{1}\right)=a_{n}^{2} P_{n}^{(\alpha, \beta)}\left(t_{0}\right) P_{n}^{(\alpha, \beta)}\left(t_{1}\right)
$$

with $t_{0}, t_{1}$ given in (7.3), (7.4), respectively. Moreover, external ellipsoidal $h$-harmonics assume the form

$$
\mathcal{F}_{n, \mathbf{0}}\left(x_{0}, x_{1}\right)=a_{n} b_{n} Q_{n}^{(\alpha, \beta)}\left(t_{0}\right) P_{n}^{(\alpha, \beta)}\left(t_{1}\right) .
$$

Formula (7.5) is meaningful for all $\left(x_{0}, x_{1}\right)$ which do not lie on the common focal line $-\sqrt{2} \leq$ $x_{0} \leq \sqrt{2}, x_{1}=0$ of the confocal ellipses determined by a constant value of the ellipsoidal coordinate $t_{0}$.

Formula (6.5) now expresses the function (7.5) in terms of the function (7.2) as follows.

Theorem 7. Let $n \in \mathbb{N}_{0}$. For every $\left(x_{0}, x_{1}\right) \in \mathbb{R}^{2}$ with $x_{0}^{2}+x_{1}^{2}>2$ we have

$$
\begin{aligned}
b_{n} Q_{n}^{(\alpha, \beta)} & \left(t_{0}\right) P_{n}^{(\alpha, \beta)}\left(t_{1}\right) \\
& =\sum_{r=0}^{\infty} \frac{(-1)^{r}}{2^{2 r} r !(2 n+\alpha+\beta+2)_{r}}\left(\mathcal{D}_{1}^{2}-\mathcal{D}_{0}^{2}\right)^{r}\left(x_{0}^{2}+x_{1}^{2}\right)^{-n-\alpha-\beta-1} P_{n}^{(\alpha, \beta)}\left(\frac{x_{0}^{2}-x_{1}^{2}}{x_{0}^{2}+x_{1}^{2}}\right),
\end{aligned}
$$

where on the left-hand side $t_{0}, t_{1}$ are given by (7.3), (7.4), respectively.

Obviously, we could replace $\mathcal{D}_{1}^{2}-\mathcal{D}_{0}^{2}$ by $2 \mathcal{D}_{1}^{2}$ on the right-hand side of (7.6). Formula (7.6) expands the left-hand side in a series of homogeneous functions whose degree decreases with $r$. The convergence will be especially good when $x_{0}^{2}+x_{1}^{2}$ is large. The formula can be checked with symbolic computer algebra by replacing $x_{j}$ by $u x_{j}$ on the left-hand side and then expanding in an asymptotic series at $u=\infty$. Computer algebra confirmed the correctness of (7.6) for small values of $n$. The natural question arises whether this formula or the more general formula (6.5) can be proved more directly, that is, without using any integrals in the proof.

\section{References}

[1] Arscott F.M., Periodic differential equations. An introduction to Mathieu, Lamé, and allied functions, International Series of Monographs in Pure and Applied Mathematics, Vol. 66, A Pergamon Press Book, The Macmillan Co., New York, 1964.

[2] Ben Saïd S., Ørsted B., The wave equation for Dunkl operators, Indag. Math. (N.S.) 16 (2005), 351-391.

[3] Blimke J., Myklebust J., Volkmer H., Merrill S., Four-shell ellipsoidal model employing multipole expansion in ellipsoidal coordinates, Med. Biol. Eng. Comput. 46 (2008), 859-869.

[4] Dassios G., The magnetic potential for the ellipsoidal MEG problem, J. Comput. Math. 25 (2007), $145-156$. 
[5] Dassios G., Kariotou F., Magnetoencephalography in ellipsoidal geometry, J. Math. Phys. 44 (2003), 220241.

[6] de Jeu M.F.E., The Dunkl transform, Invent. Math. 113 (1993), 147-162.

[7] Dunkl C.F., Reflection groups and orthogonal polynomials on the sphere, Math. Z. 197 (1988), 33-60.

[8] Dunkl C.F., A Laguerre polynomial orthogonality and the hydrogen atom, Anal. Appl. (Singap.) 1 (2003), 177-188, math-ph/0011021.

[9] Dunkl C.F., Xu Y., Orthogonal polynomials of several variables, Encyclopedia of Mathematics and Its Applications, Vol. 81, Cambridge University Press, Cambridge, 2001.

[10] Evans L., Partial differential equations, Graduate Studies in Mathematics, Vol. 19, American Mathematical Society, Providence, RI, 1998.

[11] Heine E., Handbuch der Kugelfunktionen, Vol. 1, G. Reimer Verlag, Berlin, 1878.

[12] Heine E., Handbuch der Kugelfunktionen, Vol. 2, G. Reimer Verlag, Berlin, 1881.

[13] Hikami K., Boundary $K$-matrix, elliptic Dunkl operator and quantum many-body systems, J. Phys. A: Math. Gen. 29 (1996), 2135-2147.

[14] Hobson E.W., The theory of spherical and ellipsoidal harmonics, Cambridge University Press, Cambridge, 1931.

[15] Kalnins E.G., Miller W. Jr., Jacobi elliptic coordinates, functions of Heun and Lamé type and the Niven transform, Regul. Chaotic Dyn. 10 (2005), 487-508.

[16] Niven W.D., On ellipsoidal harmonics, Phil. Trans. Royal Society London A 182 (1891), 231-278.

[17] Rösler M., Dunkl operators: theory and applications, in Orthogonal Polynomials and Special Functions (Leuven, 2002), Lecture Notes in Math., Vol. 1817, Springer, Berlin, 2003, 93-135, math.CA/0210366.

[18] Rösler M., Voit M., Markov processes related with Dunkl operators, Adv. in Appl. Math. 21 (1998), 575-643.

[19] Stieltjes T.J., Sur certains polynômes qui vérifient une équation différentielle linéaire du second ordre et sur la théorie des fonctions de Lamé, Acta Math. 5 (1885), 321-326.

[20] Szegö G., Orthogonal polynomials, 4th ed., American Mathematical Society, Providence, R.I., 1975.

[21] Volkmer H., Generalized ellipsoidal and sphero-conal harmonics, SIGMA 2 (2006), 071, 16 pages, math.CA/0610718.

[22] Whittaker E.T., Watson G.N., A course in modern analysis, Cambridge University Press, Cambridge, 1927.

[23] Xu Y., Orthogonal polynomials for a family of product weight functions on the spheres, Canad. J. Math. 49 (1997), 175-192. 